\title{
TAGUNG
}

\section{Lehren einer flexiblen Integrationspolitik: Das Verhältnis der Schweiz zur Europäischen Union}

\author{
Ilja Baudisch und Ann Gäbler*
}

Peter-Christian Müller-Graff erläuterte in seiner Einführung, dass die Schweiz als erste liberale und vielsprachige demokratische Föderation einerseits ein starkes Vorbild für ein gedeihliches Zusammenleben darstelle, andererseits aber nicht unmittelbar an der Entwicklung der Europäischen Union teilnehme. Daraus ergebe sich eine wechselseitige Herausforderung: Die EU müsse sich selbst in politischer, ökonomischer, rechtlicher und kultureller Hinsicht fragen, weshalb die Schweiz eine eigene Unionsmitgliedschaft bislang verweigere. Aber auch die Schweiz, welche ihrerseits durch das Gemeinschaftsrecht stark beeinflusst werde, müsse überdenken, ob der autonome Nachvollzug europäischer Rechtsakte ohne eigene Mitgliedschaft und ohne Beteiligung am Binnenmarkt ökonomisch und politisch auch in Zukunft vorteilhaft sei. Insoweit warb Müller-Graff für eine Europapolitik aller Möglichkeiten.

Die bestehenden Rechtsbeziehungen zwischen der Schweiz und der EU

Christa Tobler beschäftigte sich mit dem acquis der vertraglichen Verbindungen der Schweiz zur Europäischen Union und Europäischen Gemeinschaft. Tobler zeichnete dabei das Bild eines sehr komplexen und unübersichtlichen Geflechtes von bilateralen Verträgen, die der Schweiz einen Zugang zum Binnenmarkt ohne eigene EU-Mitgliedschaft ermöglichen. Ihre These, dass auch Jahrzehnte nach Inkrafttreten des Freihandelsabkommens und vier Jahre nach Inkrafttreten der Bilatera-

\section{Die Schweiz und Europa}

8. Gemeinsame Tagung des Arbeitskreises Europäische Integration, der ECSA-Schweiz und der ECSA-Österreich

Heidelberg, 30. Juni bis 1. Juli 2006

Wissenschaftliche Leitung:

Prof. Dr. Fritz BREUSS, Wirtschaftsuniversität Wien

Prof. Dr. Thomas COTTIER, Universität Bern

Prof. Dr. Dr. h.c. Peter-Christian MÜLLER-

GRAFF, Universität Heidelberg

Begrüßung und Einführung in die Thematik

Prof. Dr. Dr. h.c. Peter-Christian MÜLLER-GRAFF

\section{Grundlagen}

Der Acquis der rechtlichen Verbindung der Schweiz zur Europäischen Gemeinschaft und Europäischen Union

Prof. Dr. Christa TOBLER, Universität Basel

Schweizer Spezifika: Direkte Demokratie, Konkordanz und Neutralität als politische Gestaltungsfaktoren

Prof. Dr. Thomas COTTIER

Österreich und Schweiz - Ökonomische Erfahrungen mit und ohne Mitgliedschaft in der Europäischen Union

Prof. Dr. Fritz BREUSS

Szenarien der Schweizer Europapolitik

Nationalrätin Christa MARKWALDER BÄR, Bern

Szenarien der Schweizpolitik der Europäischen Union

Dr. Burkard STEPPACHER, Konrad-AdenauerStiftung, St. Augustin

Einzelfragen des bilateralen Verhältnisses

Freizügigkeit

Prof. Dr. Christine KADDOUS, Universität Genf

Auswirkungen der WTO-Mitgliedschaft der Schweiz und der EG auf das bilaterale Verhältnis Dr. Matthias OESCH, Universität Bern

Das Dreiecksverhältnis Schweiz-Europäische Union-WTO im Agrarbereich

Prof. Dr. Richard SENTI, Eidgenössische Technische Hochschule Zürich

* Ilja Baudisch, wissenschaftlicher Mitarbeiter, Institut für deutsches und europäisches Gesellschafts- und Wirtschaftsrecht, Universität Heidelberg.

Ann Gäbler, wissenschaftliche Mitarbeiterin, Institut für deutsches und europäisches Gesellschafts- und Wirtschaftsrecht, Universität Heidelberg. 
len I in wichtigen Punkten noch unklar sei, was eigentlich zum acquis gehöre, belegte Tobler anhand ausgewählter Einzelaspekte, unter anderem am Beispiel des „Champagner"Streitfalles im Zusammenhang mit dem Landwirtschaftsabkommen von 1999.

Anschließend beleuchtete Thomas Cottier die tief im Schweizer Nationalbewusstsein verankerten, spezifischen politischen Gestaltungsfaktoren der direkten Demokratie, der Konkordanz, des Föderalismus und der Neutralität, die zu dem Paradoxon führten, dass die Schweiz zwar eine der am stärksten integrierten Volkswirtschaften Europas sei und unter massivem Einfluss des Gemeinschaftsrechts stehe, ohne dabei jedoch Mitglied in der EU zu sein. Problematisch sei etwa die Vereinbarkeit eines Unionsbeitritts mit der Schweizer Verhandlungsdemokratie, wenngleich sich hier ein stiller, aber dynamischer Verfassungswandel abzeichne. Cottier betonte daher die Möglichkeiten einer differenzierten Integration, die den traditionellen Gestaltungsfaktoren der Schweiz am ehesten entspreche.

Fritz Breuss wandte sich den ökonomischen Aspekten der Nichtmitgliedschaft der Schweiz in der Europäischen Union zu, wobei er Österreich zum Vergleich heranzog. Sowohl der EU-Beitritt Österreichs als auch die Nichtmitgliedschaft der Schweiz hätten jeweils Vor- und Nachteile. Breuss kam zu dem Ergebnis, dass sich die Mitgliedschaft in der EU für Österreich in wirtschaftlicher Sicht insgesamt positiv ausgewirkt habe, wofür auch die Osterweiterung ausschlaggebend gewesen sei. Für die Schweiz sei die bilaterale Integrationspolitik hingegen ökonomisch neutral; die ausgebliebenen negativen Folgen einer EU-Mitgliedschaft würden die gleichfalls verhinderten positiven Effekte eines unbeschränkten Zugangs zum europäischen Binnenmarkt sehr wahrscheinlich vollständig ausgleichen.

Christa Markwalder Bär stellte in ihrem Vortrag die möglichen europapolitischen Optio-

\section{Perspektiven}

Querverbindungen bzw. „Harmonisierung“ des schweizerischen mit dem europäischen Kartellrecht

Prof. Dr. Roger ZÄCH, Universität Zürisch

Die Umsetzung der Bilateralen in den Kantonen PD Dr. Stephan KUX, Universität Zürich

Die Eidgenossenschaft als Vor- und Leitbild für die Einigung Europas

Prof. Dr. Heinrich SCHNEIDER, Universität Wien

Die privilegierte Partnerschaft der Schweiz als Modell für die Erweiterungsfrage der Europäischen Union

Prof. Dr. Christian CALLIESS, Universität Göttingen

Flexible Integration von Drittstaaten im Vergleich Prof. Dr. Sandra LAVENEX, Universität Luzern

Schlussbetrachtung: Das Autonomieparadox der Schweiz in der europäischen Integration

Prof. Dr. Dr. h.c. Peter-Christian MÜLLERGRAFF

nen der Schweiz vor. Ein Schweizer Alleingang durch Kündigung der bilateralen Verträge müsse auf jeden Fall wegen der damit verbundenen politischen Isolation und wirtschaftlichen Desintegration vermieden werden. Die langfristige Beibehaltung des Status Quo beziehungsweise eine Beteiligung der Schweiz am Europäischen Wirtschaftsraum lehnte Markwalder Bär ebenso wie ein Rahmenabkommen oder eine Zollunion als für die Lösung der europarechtlichen Probleme der Schweiz ungeeignet ab. Stattdessen favorisierte sie die EU-Vollmitgliedschaft der Schweiz, auch wenn diese kurzfristig nicht realisierbar sei.

Burkard Steppacher widmete sich der Frage nach einer europäischen Schweizpolitik. Aus europäischer Sicht zeige sich die Schweiz vor allem als ein wohlhabender und neutraler Staat, dessen historische, politische und kulturelle Besonderheiten einer engeren institutionellen Beziehung zur EU eher hinderlich seien. Die Gemeinschaft sei in der Vergangenheit aber stets bereit gewesen, die Schweiz auf bilateralem Wege unterhalb eines EUBeitritts in ihre Politik einzubeziehen. Dies resultiere möglicherweise daraus, dass die Schweiz aus EU-Sicht weder als politisches noch als wirtschaftliches Problem wahrge- 
nommen werde und zudem kein einheitliches Meinungsbild zur Schweiz existiere. Durch die Zugeständnisse der EU sei allerdings ein schweizerischer EU-Beitritt nunmehr in weitere Ferne gerückt. Abschließend zeigte Steppacher verschiedene Szenarien der Schweizpolitik auf und zog mittelfristig die Fortsetzung des Bilateralismus oder den Abschluss eines Rahmenabkommens mit ergänzendem politischem Dialog in Betracht.

Bewertung der ,Bilateralen" in der Rechtspraxis

Christine Caddous stellte in ihrem Vortrag das zwischen der Schweiz und der Europäischen Gemeinschaft und ihren Mitgliedstaaten geschlossene Freizügigkeitsabkommen vor, um die mit der Praxis bilateraler Verträge regelmäßig verbundenen Probleme aufzuzeigen. Als völkerrechtlicher Vertrag, der auch Fragen des Ausländerrechts berühre, stelle das Abkommen keine vollständige Übernahme des acquis communautaire dar, lehne sich aber stark an dessen Inhalt an; Divergenzen $\mathrm{zu}$ freizügigkeitsspezifischen EG-Regelungen bestünden daher nur in Einzelfragen. Problematisch sei hingegen die mit Art. 16 Abs. 2 des Abkommens erzeugte Statik, indem nur die bis zur Unterzeichnung ergangene EuGH-Rechtsprechung verbindlich einbezogen sei. Trotz der Ermächtigung, die Auswirkungen späterer Urteile verbindlich festzustellen, sind Unterschiede ab dem 21.6.1999 zumindest möglich. Auch Änderungen des Gemeinschaftsrechts könnten Schwierigkeiten erzeugen. So hatte etwa der Erlass der RL 2004/38/EG, die Lebenspartner auf europäischer Ebene als Familienangehörige klassifiziert, für das Freizügigkeitsabkommen keine unmittelbare Wirkung.

Im nächsten Beitrag befasste sich Matthias Oesch mit der Frage, welche Auswirkungen die WTO-Mitgliedschaft der Schweiz und der Europäischen Gemeinschaft auf das bilaterale Verhältnis hat. Zunächst erörterte er die Voraussetzungen, unter denen eine von den Prinzipien des WTO-Rechts abweichende bi- laterale Vereinbarung möglich ist. Danach sind integrationstiefere Regionalverbindungen grundsätzlich zulässig, wenn die Voraussetzungen der Art. XXIV GATT 1994 beziehungsweise Art. V GATS erfüllt sind. Für die im Freihandelsabkommen ausgeklammerten Sektoren Landwirtschaft und Dienstleistung sind nach Oesch diese Ausnahmen nicht gegeben, so dass auf präferentielle bilaterale Abkommen entweder zu verzichten oder eine umfassende Freihandelszone beziehungsweise ein Dienstleistungsabkommen gemäß den WTO-Anforderungen anzustreben ist. Zum Abschluss erläuterte er die Möglichkeiten einer Streitbeilegung nach WTO-Recht, wenn dazu im bilateralen Verhältnis eine Regelung fehlt.

Den ersten Konferenztag schloss Richard Senti mit dem Referat zum Verhältnis der Schweiz, der EG und der WTO im Agrarbereich ab. Er verwies darauf, dass die Schweiz ihre Landwirtschaft sowohl im Inneren als auch nach außen in besonderem Maße schütze. So sei eine Beteiligung am GATT im Bereich der Landwirtschaft ausgeblieben, und auch das Freihandelsabkommen mit der EG klammere landwirtschaftliche Produkte aus, während die Subventionen weiter stetig zunehmen würden. Dies habe maßgeblich zum Verlust der Wettbewerbsfähigkeit der landwirtschaftlichen Unternehmen in der Schweiz geführt. Senti zeigte auf, welche Veränderungen zur Schaffung einer wettbewerbsfähigen Schweizer Landwirtschaft nötig wären.

Ansätze zur Intensivierung und Weiterentwicklung der rechtlichen Verbindung

Über zahlreiche Parallelen, die das schweizerische mit dem europäischen Kartellrecht seit den schweizerischen Kartellgesetz-Novellen von 1995 und 2004 verbinden, berichtete Roger Zäch. Als Grund für die Aufgabe der traditionell kartellfreundlichen Politik der Schweiz und für die ,faktische "Harmonisierung mit den EG-Wettbewerbsregeln nannte Zäch den komparativen Kostenvorteil, den Länder mit funktionierendem Wettbewerbs- 
recht gegenüber der damals stark kartellierten und für ausländische Unternehmen relativ offenen Schweiz hatten. Zunächst erläuterte er die Totalrevision von 1995, die der Schweiz ein modernes Kartellgesetz verschafft, jedoch aus politischen Gründen auf Direktsanktionen verzichtet hatte. Danach ging er auf die Kartellgesetz-Novelle von 2004 und die neu eingefügte Norm des Art. 49a ein, die zur Stärkung der präventiven Wirkung für , harte Verstöße direkte Sanktionen vorsieht, den Bußgeldrahmen an die unionsübliche ZehnProzent-Grenze angleicht und eine Bonusregelung für kooperationswillige Unternehmen kennt. Ferner verwies Zäch auf die Abstützung der Kartellgesetz-Revision durch Art. 27 Bundesverfassung (BV), womit der Gesetzgeber klargestellt habe, dass neben dem Wettbewerb als Institution auch die individuelle Freiheit geschützt sei. Kritisch beurteilte er daher Tendenzen in der EG, statt auf diese Kriterien zunehmend auch auf Aspekte der wirtschaftlichen und sozialen Wohlfahrt abzustellen.

Der folgende Beitrag von Stephan Kux widmete sich der Umsetzung der Bilateralen in den Kantonen und deren Rolle in der Integrationspolitik. Festzustellen sei eine in den letzten zehn Jahren enorm gestiegene Bedeutung der kantonalen Mitwirkungsrechte (Art. 55 BV). Hintergrund dafür sei der Umstand, dass zahlreiche EU-bezogene Politikfelder auch klassische Kompetenzen der Kantone betreffen. Detailliert erläuterte Kux die Mitwirkungsinstrumente, insbesondere über die Europakommission der Konferenz der Kantonsregierungen in Bern. Dann ging er auf das als ,integration by stealth“ apostrophierte Phänomen ein, dass die verstärkte Mitwirkung weithin unbemerkt tiefe Veränderungen in der Integrationspolitik bewirkt habe. Aber auch die Abkommen hätten mehr Transformation in den Strukturen der Kantonsverwaltung erzeugt als gemeinhin wahrgenommen. Zur Lösung der daraus resultierenden Probleme schlug Kux eine institutionelle Straffung, den Abschluss eines Rahmenabkommens Bund-Kantone und eine verstärkte Zusammenarbeit auf kantonaler Ebene vor.

Anschließend untersuchte Heinrich Schneider aus historischer Perspektive, wie die Schweiz als Heimat und Herberge europäisch-föderalen Denkens die Ideen der Integration beeinflusst hat. Schneider zufolge wurde die „Helvetisierung“ gegenüber der „Balkanisierung“ als Leitbild eines bürgerschaftlichen Gemeinwesens verstanden; noch vor dem Weltkrieg fanden auf schweizerischem Boden erste konzeptionelle Vorarbeiten für die spätere Europapolitik statt. Er verwies zum einen auf die Leistungen vieler Einzelpersönlichkeiten, zum Beispiel Denis de Rougemont, Ernst von Schenck und Max Imboden; zum anderen auf Organisationen wie die EuropaUnion, die Union Européene des Fédéralistes oder die Europäische Parlamentarische Union. Insgesamt habe die Schweiz stets ein gutes Beispiel einer funktionierenden multilingualen, demokratischen Föderation gegeben und mit ihrer Neutralität als Vorbild für eine friedliche dritte Kraft in Europa gedient.

Christian Calliess widmete sich dem Konzept der privilegierten Partnerschaft und warf die Frage auf, ob die Schweizer Anbindung modellhaft für andere Staaten sein könne. Als erstes schilderte er die in den Verträgen angelegte immer engere Integration als Gefahr des „imperial overstretch“ und der Rückentwicklung zur rein ökonomischen Verbindung. Der folgenden Diskussion des Begriffs der, privilegierten Partnerschaft' mit Blick auf die Türkei schloss er einen Vergleich mit der Schweiz (und ähnlich: Norwegen) an. Dabei sei die Schweiz insofern privilegiert, als ihr mehr Freiheiten und über ein bloßes Freihandelsabkommen hinausgehende Vorteile gewährt würden. Die Übertragung auf andere Staaten sei aber schwierig, weil die schweizerische Nichtmitgliedschaft selbst gewählt sei. Alternativ schlug Calliess als neuen Anbindungsstatus eine ,flexible Mitgliedschaft“ ohne Anspruch auf Vollmitgliedschaft vor. 
Sandra Lavenex untersuchte die Assoziierungsabkommen als Form neuer „weicher“ Steuerungsmodelle auf ihre Eignung für eine flexible Integrationspolitik. Dabei unterschied sie auf makroinstitutioneller beziehungsweise sektoraler Ebene verschiedene Steuerungsmodi und stellte fest, dass in hierarchischen und in kooperativen Modellen institutionelle Grenzen prinzipiell aufrecht erhalten blieben, während in Netzwerken eine Verschiebung möglich sei. Als einen Sonderfall der Assoziierungszusammenarbeit erläuterte sie das „Comix“-Modell. Künftige Herausforderungen sah sie in der Untersuchung der Wechselwirkungen zwischen supranationaler (hierarchischer) Governance und Netzwerkgovernance.
Die Tagungsergebnisse fasste Peter-Christian Müller-Graff zusammen und betonte das aus seiner Sicht fortbestehende Autonomieparadoxon: Die Autonomie der Schweiz sei hinzunehmen, falls die Schweiz durch fortgesetzte Anpassung ihre prinzipielle Mitgliedschaftsfähigkeit bewahre. Klärungsbedürftig sei dagegen die Überlegenheit des EWR-Abkommens gegenüber der bilateralen Verbindung der Schweiz zur EU. Der Vorteil der bilateralen Lösung liege zwar in der Wahrung wichtiger Belange der Schweiz, bedürfe aber einer regelmäßigen Anpassung an sich wandelnde Umstände. Eindringlich warnte Müller-Graff davor, die Bilateralen als bloßes wirtschaftsvölkerrechtliches Abkommen zu verstehen.

\section{Neu im Programm}

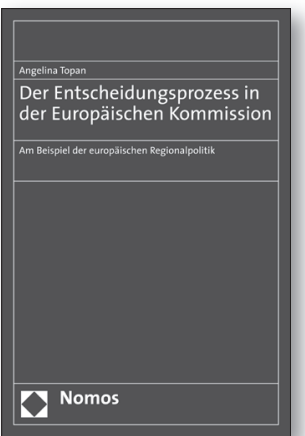

\section{Der Entscheidungsprozess in der Europäischen Kommission} Am Beispiel der europäischen Regionalpolitik Von Angelina Topan

2006, 299 S., brosch., 59,- $€$, ISBN 978-3-8329-2182-8

Der Band trägt zur Transparenz des Entscheidungsprozesses in der Europäischen Kommission bei und gibt gleichzeitig Entscheidungsträgern strategische Hinweise geben, wie sie Themen auf die Agenda der Kommission platzieren können. 